\title{
Disjoint direct product decomposition of permutation groups
}

\author{
Mun See Chang Christopher Jefferson
}

May 14, 2021

\begin{abstract}
Let $H \leq S_{n}$ be an intransitive group with orbits $\Omega_{1}, \Omega_{2}, \ldots, \Omega_{k}$. Then certainly $H$ is a subdirect product of the direct product of its projections on each orbit, $\left.H\right|_{\Omega_{1}} \times\left. H\right|_{\Omega_{2}} \times \ldots \times$ $\left.H\right|_{\Omega_{k}}$. Here we provide a polynomial time algorithm for computing the finest partition $P$ of the $H$-orbits such that $H=\left.\prod_{c \in P} H\right|_{c}$ and we demonstrate its usefulness in some applications.
\end{abstract}

\section{Introduction}

A direct product decomposition of a given group $H$ is an expression of $H$ as a direct product of groups. The direct product decomposition is useful for understanding the structure of the group, and to solve problems more efficiently. Hence, it is important to find an efficient algorithm for computing such decompositions. Kayal and Nezhmetdinovm, in [KN09], provided a polynomial time algorithm for computing a direct product decomposition of a group $H$ given by its multiplication table, which has input size $|H|^{2}$. We consider computing direct product decompositions of permutation groups given by generating sets, which are usually much smaller than the order of the group. Wilson, in [Wil12] gives a polynomial time solution to such a problem. However, as far as we know, this algorithm has not yet been implemented.

In this paper, we consider a particular type of direct product decomposition for finite permutation groups, which we call a disjoint direct product decomposition, which are direct product decompositions of permutation groups where the factors move disjoint sets of points, and so have disjoint supports (See Definition 1.1). We give a polynomial time algorithm for finding disjoint direct product decompositions of a permutation group given by a generating set and also demonstrate the practical efficiency of our algorithm. In this paper, we regard the direct product of permutation groups with disjoint supports as a subgroup of the symmetric group on the union of the supports of the direct factors.

Definition 1.1 (Disjoint direct product decomposition). Let $H \leq S_{n}$. We say that $H=$ $H_{1} \times H_{2} \times \ldots \times H_{r}$ is a disjoint direct product decomposition of $H$ if it is a direct product decomposition of $H$ and the groups $H_{i}$ have pairwise disjoint supports. Each factor $H_{i}$ is called a disjoint direct factor of $H$, which can be identified as a subgroup of $H$ that fixes all points outside $\operatorname{Supp}\left(H_{i}\right)$.

If there exists a disjoint direct product decomposition $H=H_{1} \times H_{2} \times \ldots \times H_{r}$ of $H$ with $r>1$, then we say that $H$ is d.d.p. decomposable, otherwise we say that $H$ is d.d.p. indecomposable. A disjoint direct product decomposition is finest if each factor is d.d.p. indecomposable.

Since the disjoint direct product decomposition is more restrictive than a more general decomposition, it can be computed much faster and has many useful applications. As we 
will demonstrate in Section 5, the disjoint direct product decomposition of a permutation group can be used to greatly speed up various other calculations with permutation groups, such as computing the derived subgroup, number of conjugacy classes and a composition series of a given permutation group. Furthermore, calculations that previously could not be completed in a reasonable time frame can be solved very quickly using the disjoint direct product decomposition to subdivide the computation into smaller pieces.

Another important application of disjoint direct product decompositions lies in other areas of computer science, where groups arise from symmetries of combinatorial objects. To reduce the computation time, groups are used to eliminate the symmetries of the objects through a process called symmetry breaking [GPP06].

Donaldson et al. [DM06] use the disjoint direct product decomposition to improve the performance of detecting symmetric states in model checking and Grayland et. al. [GJMRD09, Theorem 10] uses the disjoint direct product decomposition when generating symmetry breaking constraints for symmetric problems. Grayland et. al. give an algorithm for symmetry breaking which uses the disjoint direct product of two symmetry groups [GJMRD09] but otherwise does not consider general direct product decompositions. In both of these applications, disjoint direct product decompositions lead to significant speed-ups.

For these applications, the time saved depends on the number of factors in the decomposition. Hence we are interested in an algorithm that always computes a finest disjoint direct product decomposition. By the Krull-Schmidt theorem, any finite group has a unique finest direct product decomposition [Hun74, Theorem 3.8]. In Proposition 3.2, we show that the finest disjoint direct product decomposition of a given finite permutation group is unique.

The main result of this paper is to provide an efficient algorithm to compute the finest disjoint direct product decomposition of a given permutation group, and hence to prove the following:

Theorem 1.2. Let $H \leq S_{n}$ be given by a generating set $X$. Then the finest disjoint direct product decomposition of $H$ can be computed in time polynomial in $n \cdot|X|$.

Our algorithm behind Theorem 1.2 manipulates a strong generating set and therefore is fast in practice once a base and strong generating set have been found. Finding a base and strong generating set is an initial part of most permutation group algorithms. Hence, finding a disjoint direct product decomposition will not add significantly to the runtime of these algorithms.

The structure of this paper is as follows. In Section 2, we present some related work in the literature, and the definitions, notation and background knowledge we use later on. In Section 3, we present the theoretical framework which we use for the algorithms we present in Section 4. Also in Section 4, we prove Theorem 1.2. Lastly, in Section 5, we demonstrate how the algorithm can be used to speed up computation in various permutation group theoretic functions in GAP.

\section{Background and preliminaries}

If $G$ is a direct factor of $H$, then $G \unlhd H$. So a naive approach to finding its disjoint direct product decomposition is to consider all normal subgroups $N$ of $H$, check if there exists $K$ such that $N \times K=H$, then recursively try to decompose $N$ and $K$. While it is possible to optimise this approach, it has worst-case exponential complexity, since it requires considering all normal subgroups of $H$, the number of which grows exponentially with $|\operatorname{Supp}(H)|$. 
Wilson's polynomial time algorithm in [Wil12] computes the finest (not necessarily disjoint) direct product decomposition of a given permutation group $H$. As far as we are aware, the algorithm has yet to be implemented. We show that it is substantially easier to compute the finest disjoint direct product decomposition than the finest direct product decomposition.

Donaldson and Miller in [DM09, Section 5.1] present a polynomial algorithm for computing a disjoint direct factor decomposition by considering the given generators. They used the observation that, if $H=\langle X\rangle$ and there exists $S \varsubsetneqq X$ such that the support of $S$ and the support of $X \backslash S$ are disjoint, then $H=\langle S\rangle \times\langle X \backslash S\rangle$ is a disjoint direct product decomposition. The method by Donaldson and Miller is a subprocedure of the algorithm we present in Section 4. However, note that the method in [DM09] does not guarantee that the decomposition is the finest possible as different choices of $X$ may produce different decompositions. Donaldson and Miller reported that, using the generators computed from the graph automorphism program they used, this method seems to almost always produce the finest decomposition. We hypothesise that these programs almost always produce separable strong generating sets, which we shall define in Definition 3.10.

In [DM09], Donaldson and Miller also present an exponential-time algorithm to compute the finest disjoint direct product decomposition of $H$. The algorithm involves recursively computing disjoint direct product decompositions with two factors. To construct such a decomposition of $H$, they consider all partitions of the set of $H$-orbits with two cells. They test if each partition gives rise to a disjoint direct product decomposition by checking if $H$ is the direct product of its restriction to the union of orbits in the first cell and its restriction to the union of orbits in the second cell. They also made a significant improvement to their algorithm by first considering the restrictions onto pairs of orbits and deciding if they are d.d.p. decomposable.

\subsection{Notation}

Throughout the paper let $\Omega$ be a finite set. Let $\bar{i}$ denote the set $\{1,2, \ldots, i\}$. Let $G \leq \operatorname{Sym}(\Omega)$. For a subset $\Delta \subseteq \Omega$, we denote by $G_{(\Delta)}$ the pointwise stabiliser of $\Delta$ in $G$. We denote by $\operatorname{Supp}(G)$ the support of $G$.

Definition 2.1. Let $G_{1}, G_{2}, \ldots, G_{k}$ be groups and let $G=G_{1} \times G_{2} \times \ldots \times G_{k}$. A subdirect product of $G$ is a subgroup $K \leq G$ such that each projection $\rho_{i}: K \rightarrow G_{i}$ onto the $i$-th factor is surjective. That is, $\rho_{i}(K)=G_{i}$ for all $1 \leq i \leq k$.

We would like to draw the reader's attention to an elementary result, which we shall repeatedly use later.

Lemma 2.2. Let $H$ be a subdirect product of $G_{1} \times G_{2}$. Then $H=G_{1} \times G_{2}$ if and only if $1 \times G_{2} \leq H$.

Proof. The forward implication is clear. For the backward implication, for all $\left(g_{1}, g_{2}\right) \in H$, since $\left(1, g_{2}\right) \in H$, we have $\left(g_{1}, 1\right) \in H$. The projection of $H$ onto $G_{1}$ is the whole $G_{1}$, so $G_{1} \times 1 \leq H$. Now since $G_{1} \times 1$ and $1 \times G_{2}$ generate $G_{1} \times G_{2}$ and are both contained in $H$, we have $H=G_{1} \times G_{2}$.

Theorem 2.3 is commonly known as Goursat's lemma. It describes the subgroups of a direct product of groups and appears in the literature in various places, including, for example, [Sch94, PS18]. Here we are only concerned about subgroups of direct products that are also 
subdirect products. However, note that every subgroup of a direct product is a subdirect product of the direct product of the images under the projections.

Theorem 2.3 ([Gou89]). Let $G_{1}, G_{2}$ be groups. Let $H$ be a subdirect product of $G_{1} \times G_{2}$. Let $\rho_{1}: H \rightarrow G_{1}$ and $\rho_{2}: H \rightarrow G_{2}$ be the projection maps of $H$ onto $G_{1}$ and $G_{2}$ respectively. The following hold.

1. Let $N_{1}:=\rho_{1}\left(\operatorname{Ker}\left(\rho_{2}\right)\right)$ and $N_{2}:=\rho_{2}\left(\operatorname{Ker}\left(\rho_{1}\right)\right)$. Then $N_{1} \unlhd G_{1}$ and $N_{2} \unlhd G_{2}$.

2. The map $\theta: G_{1} / N_{1} \rightarrow G_{2} / N_{2}$ given by $N_{1} h_{1} \mapsto N_{2} h_{2}$ if $\left(h_{1}, h_{2}\right) \in H$ is an isomorphism.

Let $T_{1}$ and $T_{2}$ be transversals of $N_{1}$ in $G_{1}$ and $N_{2}$ in $G_{2}$ respectively. Let $\hat{\theta}: T_{1} \rightarrow T_{2}$ be a map induced by $\theta$, where $\hat{\theta}\left(t_{1}\right)=t_{2}$ if $\theta\left(N_{1} t_{1}\right)=N_{2} t_{2}$. One can show that $\hat{\theta}$ is a well-defined, and letting $\mathcal{G}=\left\{\left(t_{1}, \hat{\theta}\left(t_{1}\right)\right) \mid t_{1} \in T_{1}\right\}$ be the graph of $\hat{\theta}$, we have $H=\left\langle\mathcal{G}, N_{1} \times 1,1 \times N_{2}\right\rangle$.

For subdirect products of the direct product of more than two groups, we use an asymmetrical version of Theorem 2.3, where $\theta: G_{1} \rightarrow G_{2} / N_{2}$ defined by $h_{1} \mapsto N_{2} h_{2}$ for all $\left(h_{1}, h_{2}\right) \in H$ is a surjective homomorphism. Similarly, letting $\hat{\theta}: G_{1} \rightarrow T_{2}$ be defined by $\hat{\theta}\left(g_{1}\right)=t_{2}$ if $\theta\left(g_{1}\right)=N_{2} t_{2}$ and $\mathcal{G}=\left\{\left(g_{1}, \hat{\theta}\left(g_{1}\right)\right) \mid g_{1} \in G_{1}\right\}$ be the graph of $\hat{\theta}$, we get $H=\left\langle\mathcal{G}, 1 \times N_{2}\right\rangle$. For more details, see [BSZ15, Theorem 2.3].

We will be applying Goursat's lemma to an intransitive group $H \leq S_{n}$, considered as a subdirect product of the direct product of its transitive constituents.

Let $\Delta$ be a union of (some of) the $H$-orbits. For $h \in H$, we denote by $\left.h\right|_{\Delta}$ the restriction of $h$ onto $\Delta$, that is, the permutation in $\operatorname{Sym}(\Delta)$ such that $\alpha^{\left(\left.h\right|_{\Delta}\right)}=\alpha^{h}$ for all $\alpha \in \Delta$. We denote by $\left.H\right|_{\Delta}$ the restriction of $H$ onto $\Delta$, so $\left.H\right|_{\Delta}=\left\{\left(\left.h\right|_{\Delta}\right) \mid h \in H\right\}$. Let $K \leq \operatorname{Sym}(\Delta)$ and let $\Gamma$ be a set disjoint to $\Delta$. Denote by $1_{\Gamma} \times K$ the subgroup of $\operatorname{Sym}(\Delta \cup \Gamma)$ with support $\Delta$ such that $\left.\left(1_{\Gamma} \times K\right)\right|_{\Delta}=K$.

Recall that we regard a direct product of groups that have disjoint supports as a subgroup of the symmetric group over the disjoint union of the supports of the factors. For the rest of the paper, we will use the following notation:

Notation 2.4. Let $H \leq S_{n}$. Fix an ordering $\Omega_{1}, \Omega_{2}, \ldots, \Omega_{k}$ on the $H$-orbits. For $1 \leq i \leq k$, let $G_{i}:=\left.H\right|_{\Omega_{i}}$. We consider $H$ as a subdirect product of $G:=G_{1} \times G_{2} \times \ldots \times G_{k} \leq S_{n}$.

For $1 \leq i \leq k$, let $\rho_{i}: G \rightarrow G_{i}$ be defined by $\left.h \mapsto h\right|_{\Omega_{i}}$. For a subset $I=\left\{I_{1}, I_{2}, \ldots, I_{r}\right\}$ of $\{1,2, \ldots, k\}$, let $P_{I}:\left.G \rightarrow H\right|_{\cup_{i \in I} \Omega_{i}}$ be defined by $\left.\left.\left.h \mapsto h\right|_{\Omega_{I_{1}}} h\right|_{\Omega_{I_{2}}} \ldots h\right|_{\Omega_{I_{r}}}$.

For all $1 \leq i \leq k$, let $\Delta_{i}:=\bigcup_{j \leq i} \Omega_{j}$.

Note that it is important that the $H$-orbits have to be in a fixed order. However, the choice of how we order them is unimportant. In our experiments, we chose to order the orbits by their smallest elements.

By iteratively considering $P_{\overline{i+1}}(H)$ as a subdirect product of $P_{\bar{i}}(H) \times \rho_{i+1}(H)$, we get Theorem 2.5, which gives the structure of intransitive groups and follows from [BSZ15, Theorem 3.2$]$.

Theorem 2.5 ([BSZ15]). Recall Notation 2.4. Then for all $1 \leq i \leq k-1$, the following hold.

1. Let $N_{i+1}:=\rho_{i+1}\left(H_{\left(\Delta_{i}\right)}\right)$. Then $N_{i+1} \unlhd G_{i+1}$.

2. Let $\theta_{i}: P_{\bar{i}}(H) \rightarrow G_{i+1} / N_{i+1}$ be defined by $P_{\bar{i}}(h) \mapsto N_{i+1} \rho_{i+1}(h)$. Then $\theta_{i}$ is a surjective homomorphism. 
3. Let $T_{i+1}$ be a transversal of $N_{i+1}$ in $G_{i+1}$ and

$$
\begin{aligned}
\varphi_{i}: P_{\bar{i}}(H) & \rightarrow \operatorname{Sym}\left(\Delta_{i+1}\right) \\
P_{\bar{i}}(h) & \mapsto P_{\bar{i}}(h) t \quad \text { if } t \in T_{i+1} \text { and } \theta_{i}\left(P_{\bar{i}}(h)\right)=N_{i+1} t .
\end{aligned}
$$

Then $P_{\overline{i+1}}(H)=\left\langle\operatorname{Im}\left(\varphi_{i}\right), 1_{\Delta_{i}} \times N_{i+1}\right\rangle$.

In this paper, we will always require that $1 \in T_{i+1}$ for all $1 \leq i \leq k-1$. Note also that the $\varphi_{i}$ are not homomorphisms in general, but this is not a problem since we do not use it in our computation.

We end the section with an example, which will be a running example throughout the paper.

Example 2.6. Let $x_{1}:=(1,2,3)(7,9,8)(10,12,11), \quad x_{2}:=(4,5,6)(7,8,9)(10,11,12)$, $x_{3}:=(5,6)(8,9)(11,12)$ and $x_{4}:=(7,8,9)(10,11,12)$. Let $H:=\left\langle x_{1}, x_{2}, x_{3}, x_{4}\right\rangle \leq S_{12}$.

Then $\Omega_{1}=\{1,2,3\}, \Omega_{2}=\{4,5,6\}, \Omega_{3}=\{7,8,9\}$ and $\Omega_{4}=\{10,11,12\}$ are the orbits of $H$. For $1 \leq i \leq 4$, let $G_{i}=\left.H\right|_{\Omega_{i}}$. Then $H$ is a subdirect product of $G_{1} \times G_{2} \times G_{3} \times G_{4}$, where we regard the direct product as a subgroup of $S_{12}$.

For all $1 \leq i \leq 4$, let $\Delta_{i}:=\bigcup_{j<i} \Omega_{j}$. Then $P_{\bar{i}}(H)=\left.H\right|_{\Delta_{i}}$ for all $i$. So $\left.H\right|_{\Delta_{2}}=\langle(1,2,3)$, $(4,5,6),(5,6)\rangle$ and $H_{\left(\Delta_{2}\right)}=\langle(7,8,9)(10,11,12)\rangle$.

Let the $N_{i}$ be as in Theorem 2.5. Then $N_{3}=\langle(7,8,9)\rangle$ is normal in $G_{3}$, and $N_{4}=1$.

Let $\theta_{2}:\left.H\right|_{\Delta_{2}} \rightarrow G_{3} / N_{3}$ be as in Theorem 2.5. Then $\theta((1,2,3))=\theta\left(\left.x_{1}\right|_{\Delta_{2}}\right)=\left.N_{3} x_{1}\right|_{\Omega_{3}}=N_{3}$. Similarly $\theta_{2}((4,5,6))=N_{3}$ and $\theta_{2}((5,6))=N_{3}(8,9)$. So $\theta_{2}$ is surjective.

Let $T_{3}:=\{(),(8,9)\}$ be a transversal of $N_{3}$ in $G_{3}$. Let $\varphi_{2}$ be as in Theorem 2.5. Then $\varphi_{2}\left(\left.H\right|_{\Delta_{2}}\right)=\langle(1,2,3),(4,5,6),(5,6)(8,9)\rangle$, and one could check that indeed $\left\langle\varphi_{2}\left(\left.H\right|_{\Delta_{2}}\right), 1_{\Delta_{2}} \times\right.$ $\left.N_{3}\right\rangle=\left.H\right|_{\Delta_{3}}$.

\section{Disjoint direct product decomposition}

Recall Notation 2.4. In this section, we will first show that $H \leq S_{n}$ has a unique finest disjoint direct product decomposition. Then in Section 3.1, we will see how the computation of disjoint direct product decompositions can be reduced to computing the $N_{i+1}$ and the kernels of the $\theta_{i}$ in Theorem 2.5. We then show that these, in turn, can be efficiently computed in Section 3.2.

Definition 3.1. We say that two disjoint direct product decompositions $H=H_{1} \times H_{2} \times \ldots \times$ $H_{r}$ and $H=K_{1} \times K_{2} \times \ldots \times K_{s}$ are equivalent if the sets of sets $\left\{\operatorname{Supp}\left(H_{i}\right) \mid 1 \leq i \leq r\right\}$ and $\left\{\operatorname{Supp}\left(K_{i}\right) \mid 1 \leq i \leq s\right\}$ are equal.

Proposition 3.2. Up to equivalence, there is a unique finest disjoint direct product decomposition of $H$.

Proof. Aiming for a contradiction, let $H=H_{1} \times H_{2} \times \ldots \times H_{r}$ and $H=K_{1} \times K_{2} \times \ldots \times K_{s}$ be two inequivalent finest disjoint direct product decompositions of $H$. Since the supports of the disjoint direct factors form a partition of $\operatorname{Supp}(H)$, there exist $1 \leq i \leq r$ and $1 \leq j \leq s$ such that $\operatorname{Supp}\left(H_{i}\right) \neq \operatorname{Supp}\left(K_{j}\right)$ and $\operatorname{Supp}\left(H_{i}\right) \cap \operatorname{Supp}\left(K_{j}\right) \neq \emptyset$. Let $\Gamma:=\operatorname{Supp}\left(H_{i}\right)$ and $\Delta:=$ $\operatorname{Supp}\left(K_{j}\right)$. We will show that $H_{i}=\left.H_{i}\right|_{\Gamma \backslash \Delta} \times\left. H_{i}\right|_{\Gamma \cap \Delta}$ is a disjoint direct product decomposition of $H_{i}$, which contradicts the fact that $H=H_{1} \times H_{2} \times \ldots \times H_{r}$ is a finest decomposition. Since $H_{i}$ is a subdirect product of $\left.H_{i}\right|_{\Gamma \backslash \Delta} \times\left. H_{i}\right|_{\Gamma \cap \Delta}$, by the backward implication of Lemma 2.2 it suffices to show that $\left.H_{i}\right|_{\Gamma \cap \Delta} \times 1_{\Gamma \backslash \Delta} \leq H_{i}$. We do so by showing that for all $h_{i} \in H_{i}$, there 
exists $h_{i}^{\prime} \in H_{i}$ such that $\left.h_{i}^{\prime}\right|_{\Gamma \cap \Delta}=\left.h_{i}\right|_{\Gamma \cap \Delta}$ and $\left.h_{i}^{\prime}\right|_{\Gamma \backslash \Delta}=1$.

Let $h_{i} \in H_{i}$. Let $\hat{h_{i}} \in S_{n}$ be such that $\left.\hat{h}_{i}\right|_{\Gamma}=h_{i}$ and $\left.\hat{h_{i}}\right|_{\bar{n} \backslash \Gamma}=1$. By the forward implication of Lemma 2.2, $\hat{h}_{i} \in H$. Similarly, since $K_{j}$ is a disjoint direct factor of $H$, there exists $h \in H$ such that $\left.h\right|_{\bar{n} \backslash \Delta}=\left.\hat{h_{i}}\right|_{\bar{n} \backslash \Delta}$ and $\left.h\right|_{\Delta}=1$. Then $h^{\prime}:=\hat{h_{i}} h^{-1}$ is an element of $H$ such that

$$
\begin{gathered}
\left.h^{\prime}\right|_{\Gamma \cap \Delta}=\left(\left.\hat{h_{i}}\right|_{\Gamma \cap \Delta}\right)\left(\left.h^{-1}\right|_{\Gamma \cap \Delta}\right)=\left.\hat{h}_{i}\right|_{\Gamma \cap \Delta}=\left.h_{i}\right|_{\Gamma \cap \Delta}, \\
\left.h^{\prime}\right|_{\Gamma \backslash \Delta}=\left(\left.\hat{h_{i}}\right|_{\Gamma \backslash \Delta}\right)\left(\left.h^{-1}\right|_{\Gamma \backslash \Delta}\right)=\left(\left.\hat{h_{i}}\right|_{\Gamma \backslash \Delta}\right)\left(\left.{\hat{h_{i}}}^{-1}\right|_{\Gamma \backslash \Delta}\right)=1, \\
\text { and }\left.h^{\prime}\right|_{\bar{n} \backslash \Gamma}=\left.\left.h^{\prime}\right|_{\Delta \backslash \Gamma} h^{\prime}\right|_{\bar{n} \backslash(\Delta \cup \Gamma)}=\left(\left.\left.\hat{h_{i}}\right|_{\Delta \backslash \Gamma} h^{-1}\right|_{\Delta \backslash \Gamma}\right)\left(\left.\left.\hat{h}_{i}\right|_{\bar{n} \backslash(\Delta \cup \Gamma)}{\hat{h_{i}}}^{-1}\right|_{\bar{n} \backslash(\Delta \cup \Gamma)}\right)=1 .
\end{gathered}
$$

Therefore, $h_{i}^{\prime}:=\left.h^{\prime}\right|_{\Gamma}$ is an element of $H_{i}$ such that $\left.h_{i}^{\prime}\right|_{\Gamma \cap \Delta}=\left.h_{i}\right|_{\Gamma \cap \Delta}$ and $\left.h_{i}^{\prime}\right|_{\Gamma \backslash \Delta}=1$.

\subsection{Computing the disjoint direct product decomposition}

Recall Notation 2.4. We will compute the finest disjoint direct product decomposition of $H$ iteratively by computing the finest disjoint direct product decomposition of $P_{\bar{i}}(H)$ for $1 \leq i \leq k$. In this subsection, we show for $1 \leq i<k$, how we can compute the finest disjoint direct product decomposition of $P_{\overline{i+1}}(H)$ using the finest disjoint direct product decomposition of $P_{\bar{i}}(H)$ and the groups $N_{i+1}$ and homomorphisms $\theta_{i}$ from Theorem 2.5.

Since the support of each disjoint direct factor of a group is a union of (some of) its orbits, we will be computing certain partitions of $\bar{i}$ for each $1 \leq i \leq k$. We will denote an unordered partition $\mathcal{P}$ of a set $\Gamma$ by $\left\langle C_{1}\left|C_{2}\right| \ldots \mid C_{r}\right\rangle$, where the $C_{i}$ are sets with disjoint intersections, called the cells of $\mathcal{P}$, and the union of the $C_{i}$ is $\Gamma$.

Definition 3.3. For $1 \leq i \leq k$, let $\mathcal{P}_{i}=\left\langle C_{1}\left|C_{2}\right| \ldots \mid C_{r}\right\rangle$ be the (unordered) partition of $\bar{i}$ consisting of cells $C_{j} \subseteq \bar{i}$ for $1 \leq j \leq r$ such that $P_{\bar{i}}(H)=P_{C_{1}}(H) \times P_{C_{2}}(H) \times \ldots \times P_{C_{r}}(H)$ is the finest disjoint direct product decomposition of $P_{\bar{i}}(H)$.

Observe that trivially, $\mathcal{P}_{1}=\langle\{1\}\rangle$. Proposition 3.4 describes how we can compute $\mathcal{P}_{i+1}$ using $\mathcal{P}_{i}$ for $1 \leq i<k$. To simplify notation, from now on, for subsets $I, J \subseteq \bar{k}$ and for $h \in H$, we denote by $P_{I}(h) \times 1_{J}$ the permutation $h^{\prime} \in \operatorname{Sym}\left(\operatorname{Supp}\left(P_{I \cup J}(H)\right)\right)$ such that $P_{I}\left(h^{\prime}\right)=P_{I}(h)$ and $P_{J}\left(h^{\prime}\right)=1$. Similarly, for $K \subseteq H$, we denote by $P_{I}(K) \times 1_{J}$ the set $\left\{P_{I}(h) \times 1_{J} \mid h \in K\right\}$.

Proposition 3.4. Let $1 \leq i<k$. Let $\mathcal{P}_{i}=\left\langle C_{1}\left|C_{2}\right| \ldots \mid C_{r}\right\rangle$ be as in Definition 3.3 and

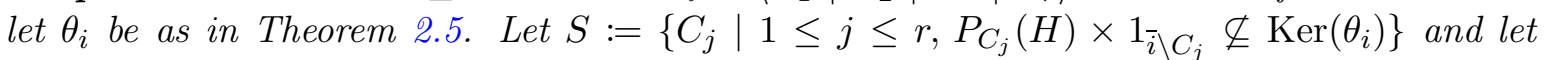
$C=\cup_{C_{j} \in S} C_{j} \cup\{i+1\} .{ }^{1}$ Then

$$
P_{\overline{i+1}}(H)=P_{C}(H) \times \prod_{C_{j} \notin S} P_{C_{j}}(H)
$$

is the finest disjoint direct product decomposition of $P_{\overline{i+1}}(H)$.

Hence the partition $\mathcal{P}_{i+1}$ from Definition 3.3 is the partition of $\overline{i+1}$ consisting of cell $C$ and all other cells $C_{j}$ of $\mathcal{P}_{i}$ such that $C_{j} \notin S$.

\footnotetext{
${ }^{1}$ Note that $S$ depends in $i$. Since it is clear from the context which $i$ the set $S$ refers to, we omit the reference to simplify notation.
} 
Proof. We will first show that Equation (1) is a disjoint direct product decomposition of $P_{\overline{i+1}}(H)$, and then we will show that it is the finest disjoint direct product decomposition. The statement on $\mathcal{P}_{i+1}$ will then follow from Definition 3.3.

Since the factors in Equation (1) move disjoint sets of points, we show that Equation (1) is a disjoint direct product decomposition of $P_{\overline{i+1}}(H)$, by showing that it gives a direct product decomposition of $P_{\overline{i+1}}(H)$. Observe that $P_{\overline{i+1}}(H)$ is a subdirect product of $P_{C}(H) \times$ $\prod_{C_{j} \notin S} P_{C_{j}}(H)$. Then by the backward implication of Lemma 2.2, it suffices to show that $1_{C} \times \prod_{C_{j} \notin S} P_{C_{j}}(H) \leq P_{\overline{i+1}}(H)$. We will do so by showing that $P_{C_{j}}(H) \times 1_{\overline{i+1} \backslash C_{j}} \leq P_{\overline{i+1}}(H)$ for all cells $C_{j}$ of $\mathcal{P}_{i}$ such that $C_{j} \notin S$, then from here it follows that Equation (1) is a disjoint direct product decomposition of $P_{\overline{i+1}}(H)$.

Let $C_{j} \notin S$. Then $\theta_{i}\left(P_{C_{j}}(H) \times 1_{\bar{i} \backslash C_{j}}\right)=N_{i+1}$. Let $\varphi_{i}$ be as in Theorem 2.5. Then $\varphi_{i}\left(P_{C_{j}}(H) \times 1_{\bar{i} \backslash C_{j}}\right)=P_{C_{j}}(H) \times 1_{\overline{i+1} \backslash C_{j}} \leq P_{\overline{i+1}}(H)$. Hence, Equation (1) gives a disjoint direct product decomposition of $P_{\overline{i+1}}(H)$.

We will now show that Equation (1) is the finest disjoint direct product decomposition. As $C_{j} \notin S$ are cells of $\mathcal{P}_{i}$, the groups $P_{C_{j}}(H)$ for $C_{j} \notin S$ are d.d.p. indecomposable, so it remains to show that $P_{C}(H)$ is d.d.p. indecomposable. Observe that for $C_{j} \notin S$, since $P_{C_{j}}(H)$ is a finest disjoint direct factor of $P_{\overline{i+1}}(H)$, each $C_{j} \notin S$ is a cell of $\mathcal{P}_{i+1}$. We proceed as follows. We first show that $C$ is the union of a subset of $U:=\left\{C_{1}, C_{2}, \ldots, C_{r},\{i+1\}\right\}$ and then show that $C_{j} \in S$ is in the same cell of $\mathcal{P}_{i+1}$ with $\{i+1\}$, from which we deduce that $C$ is a cell of $\mathcal{P}_{i+1}$ and so $P_{C}(H)$ is d.d.p. indecomposable.

To prove the first claim, we show that for all $u \in U$ such that $u \cap C \neq \emptyset$, we have $u \subseteq C$. This is trivially true for $u=\{i+1\}$. Let $C_{j}$ be a cell of $\mathcal{P}_{i}$ such that $C_{j} \cap C \neq \emptyset$. We have seen that Equation (1) is a disjoint direct product decomposition, thus the projection $P_{C}(H)$ is a disjoint direct factor of $P_{\overline{i+1}}(H)$, so $P_{C}(H) \times 1 \overline{i+1} \backslash C \leq P_{\overline{i+1}}(H)$. Applying $P_{C_{j}}$ on both sides yields $P_{C_{j} \cap C}(H) \times 1_{C_{j} \backslash C} \leq P_{C_{j}}(H)$. Since $P_{C_{j}}(H)$ is d.d.p. indecomposable, $C_{j} \cap C=C_{j}$, so $C_{j} \subseteq C$ and therefore $C$ is the union of a subset of $U$.

Lastly, we show that each $C_{j} \in S$ is in the cell of $\mathcal{P}_{i+1}$ containing $i+1$. Let $C_{j} \in S$. Aiming for a contradiction, suppose that $C_{j}$ and $i+1$ are in different cells of $\mathcal{P}_{i+1}$. Let $\varphi_{i}: P_{\bar{i}}(H) \rightarrow$ $\operatorname{Sym}\left(\Delta_{i+1}\right)$ be as in Theorem 2.5 and consider $L:=\varphi_{i}\left(P_{C_{j}}(H) \times 1_{\bar{i} \backslash C_{j}}\right) \subseteq P_{\overline{i+1}}(H)$. Let $D$ be

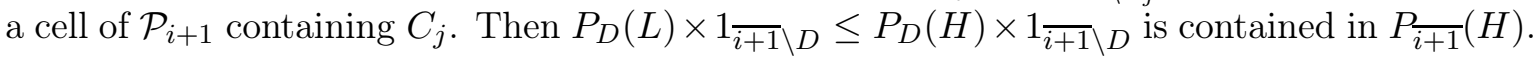
Since $i+1 \notin D$, it follows that

$$
P_{\bar{i}}(L) \times 1_{i+1}=P_{D}(L) \times 1_{\overline{i+1} \backslash D} \leq P_{\overline{i+1}}(H) .
$$

Now since both $L$ and $P_{\bar{i}}(L) \times 1_{i+1}$ are contained in $P_{\overline{i+1}}(H)$, the set $1_{\bar{i}} \times \rho_{i+1}(L)$ is also contained in $P_{\overline{i+1}}(H)$. Therefore $\rho_{i+1}(L) \subseteq N_{i+1}$ and hence $\theta_{i}\left(P_{C_{j}}(H) \times 1_{\bar{i} \backslash C_{j}}\right)=N_{i+1}$, a contradiction to the fact that $C_{j} \in S$.

Example 3.5 (running example). We return to Example 2.6. First observe that $P_{\overline{2}}(H)=\langle(1,2,3),(4,5,6),(5,6)\rangle=\rho_{1}(H) \times \rho_{2}(H)$, so $\mathcal{P}_{2}=\langle\{1\} \mid\{2\}\rangle$. We have seen from Example 2.6 that $\theta((1,2,3))=\theta_{2}((4,5,6))=N_{3}$ and $\theta_{2}((5,6))=N_{3}(8,9)$. So $\rho_{1}(H) \times 1_{\overline{3} \backslash\{1\}}=$ $\langle(1,2,3)\rangle$ is contained in $\operatorname{Ker}\left(\theta_{i}\right)$ while $\rho_{2}(H) \times 1_{\overline{3} \backslash\{2\}}=\langle(4,5,6),(5,6)\rangle$ is not contained in $\operatorname{Ker}\left(\theta_{i}\right)$. Hence $\mathcal{P}_{3}=\langle\{1\} \mid\{2,3\}\rangle$ and one can check that indeed $P_{\overline{3}}(H)=\rho_{1}(H) \times P_{\{2,3\}}(H)$.

Proposition 3.4 will be used as the core of our algorithm for finding the finest disjoint direct factor decomposition in Section 4. 


\subsection{Orbit-ordered base and separable strong generating set}

In this subsection, fix $1 \leq i<k$ and let $N_{i+1}, \theta_{i}$ and $\varphi_{i}$ be as in Theorem 2.5. We will see how we can use some fundamental data structures associated to permutation groups to compute the $N_{i+1}$ and find the cells $C_{j}$ of $\mathcal{P}_{i}$ such that $P_{C_{j}}(H) \times 1_{\bar{i} \backslash C_{j}} \not \subseteq \operatorname{Ker}\left(\theta_{i}\right)$.

We will be using a base and strong generating set, which is used in many permutation group algorithms [Sim70, Sim71]. For more information on how to find bases and strong generating sets, see [HEO05, Ser03].

Definition 3.6. A base of $H \leq S_{n}$ is a sequence $B=\left[\beta_{1}, \beta_{2}, \ldots, \beta_{m}\right]$ of points in $\bar{n}$ for some $m \in \mathbb{Z}^{+}$such that $H_{\left(\beta_{1}, \beta_{2}, \ldots, \beta_{m}\right)}=1$.

A base defines a subgroup chain $H^{[1]} \geq H^{[2]} \geq \ldots \geq H^{[m+1]}$ called a stabiliser chain, where $H^{[1]}:=H$ and $H^{[i]}:=H_{\left(\beta_{1}, \beta_{2}, \ldots, \beta_{i-1}\right)}$ for $2 \leq i \leq m+1$.

A base $B$ is non-redundant if for all $1 \leq i \leq m$, we have $H^{[i+1]} \supsetneqq H^{[i]}$. Otherwise, $B$ is said to be redundant.

A strong generating set $X$ of $H$ relative to $B$ is a generating set of $H$ where $H^{[i]}=\langle x \in X|$ $\left.x \in H^{[i]}\right\rangle$ for all $1 \leq i \leq m+1$.

Recall the $\Delta_{i}$ from Notation 2.4. We will be using bases that are orbit-ordered:

Definition 3.7. A base $B:=\left[\beta_{1}, \beta_{2}, \ldots, \beta_{m}\right]$ of $H \leq S_{n}$ is orbit-ordered with respect to the ordering $\Omega_{1}, \Omega_{2}, \ldots, \Omega_{k}$ of the $H$-orbits if there exist $1 \leq j_{1} \leq j_{2} \leq \ldots \leq j_{k} \leq m$ such that for all $1 \leq i \leq k$, we have $H_{\left(\beta_{1}, \beta_{2}, \ldots, \beta_{j_{i}}\right)}=H_{\left(\Delta_{i}\right)}$.

Remark 3.8. Recall from Notation 2.4 that we fixed an ordering $\Omega_{1}, \Omega_{2}, \ldots, \Omega_{k}$ of the $H$-orbits. Then the concatenation of $\Omega_{1}, \Omega_{2}, \ldots, \Omega_{k}$ is a (redundant) orbit-ordered base of $H$.

We can compute $N_{i+1}$ from a strong generating set of $H$ relative to an orbit-ordered base.

Lemma 3.9. Let $B:=\left[\beta_{1}, \beta_{2}, \ldots, \beta_{m}\right]$ be an orbit-ordered base of $H \leq S_{n}$ with respect to the ordering $\Omega_{1}, \Omega_{2}, \ldots, \Omega_{k}$. Let $X$ be a strong generating set of $H$ with respect to $B$. Then $N_{i+1}=\left\langle\rho_{i+1}(x) \mid x \in X \cap H_{\left(\Delta_{i}\right)}\right\rangle$ for all $1 \leq i<k$.

Proof. Since $B$ is orbit-ordered, there exists $1 \leq j \leq m$ such that $H_{\left(\beta_{1}, \beta_{2}, \ldots, \beta_{j}\right)}=H_{\left(\Delta_{i}\right)}$. As $X$ is a strong generating set relative to $B$, we have $H_{\left(\Delta_{i}\right)}=\left\langle X \cap H_{\left(\Delta_{i}\right)}\right\rangle$. The result follows since $N_{i+1}=\rho_{i+1}\left(H_{\left(\Delta_{i}\right)}\right)$.

Recall that we compute the $\mathcal{P}_{i}$ iteratively. At the $i$-th iterative step, we will produce a strong generating set for $H$ that is $i$-separable:

Definition 3.10. Let $\mathcal{P}_{i}$ be as in Definition 3.3. A strong generating set $X$ of $H$ with respect to an orbit-ordered base $B$ is $i$-separable if for all $x \in X$ with $P_{\bar{i}}(x) \neq 1$, there exists a unique cell $C_{j}$ of $\mathcal{P}_{i}$ such that $P_{\bar{i}}(x) \in P_{C_{j}}(H) \times 1_{\bar{i} \backslash C_{j}}$.

We say that $X$ is separable if it is $k$-separable, where $k$ is the number of $H$-orbits.

Note that since we have fixed an ordering of the $H$-orbits, by Proposition 3.2 , the $\mathcal{P}_{i}$ are unique, so $i$-separability depends only on $i$.

Example 3.11 (running example). Recall Example 2.6, where $x_{1}:=(1,2,3)(7,9,8)(10,12,11)$, $x_{2}:=(4,5,6)(7,8,9)(10,11,12), x_{3}:=(5,6)(8,9)(11,12)$ and $x_{4}:=(7,8,9)(10,11,12)$. We order the $H$-orbits by their smallest elements, so we have an ordering $\Omega_{1}, \Omega_{2}, \Omega_{3}, \Omega_{4}$. Then 
$B:=[1,4,5,7]$ is an orbit-ordered base since $H_{\left(\Delta_{1}\right)}=H_{(1)}, H_{\left(\Delta_{2}\right)}=H_{(1,4,5)}, H_{\left(\Delta_{3}\right)}=H_{\left(\Delta_{4}\right)}=$ $H_{(1,4,5,7)}$. The generating set $X:=\left\{x_{1}, x_{2}, x_{3}, x_{4}\right\}$ is a strong generating set of $H$ with respect to the base $B$. So $P_{\overline{2}}(H)=\langle(1,2,3),(4,5,6),(5,6)\rangle$ and hence $\mathcal{P}_{2}=\langle\{1\} \mid\{2\}\rangle$. Then $X$ is 2-separable since

$$
P_{\overline{2}}\left(x_{1}\right) \in \rho_{1}(H) \times 1_{2} \quad \text { and } \quad P_{\overline{2}}\left(x_{2}\right), P_{\overline{2}}\left(x_{3}\right) \in 1_{1} \times \rho_{2}(H) \quad \text { and } \quad P_{\overline{2}}\left(x_{4}\right)=1 .
$$

Similarly, we can show that $\mathcal{P}_{3}=\langle\{1\} \mid\{2,3\}\rangle$. The set $X$ is not 3 -separable since $\rho_{1}\left(x_{1}\right)$ and $P_{\{2,3\}}\left(x_{1}\right)$ are both non-trivial.

For each cell $C_{j}$ of $\mathcal{P}_{i}$, we may compute $\theta_{i}\left(P_{C_{j}}(H) \times 1_{\bar{i} \backslash C_{j}}\right)$ using an $i$-separable strong generating set of $H$ :

Lemma 3.12. Let $X$ be an i-separable strong generating set of $H$. Then for each cell $C_{j}$ of $\mathcal{P}_{i}$, we have

$$
\left.\theta_{i}\left(P_{C_{j}}(H) \times 1_{\bar{i} \backslash C_{j}}\right)=\left\langle N_{i+1} \rho_{i+1}(x)\right| x \in X \text { and } P_{C_{j}}(x) \neq 1\right\rangle .
$$

Proof. Since $X$ is $i$-separable, $P_{C_{j}}(H) \times 1_{\bar{i} \backslash C_{j}}$ is generated by the $P_{\bar{i}}(x)$ where $x \in X$ such that $P_{C_{j}}(x) \neq 1$. So $\theta_{i}\left(P_{C_{j}}(H) \times 1_{\bar{i} \backslash C_{j}}\right)=\left\langle N_{i+1} \rho_{i+1}(x)\right| x \in X$ and $\left.P_{C_{j}}(x) \neq 1\right\rangle$.

In our algorithm, we will be using the sifting procedure to compute an $(i+1)$-separable strong generating set from an $i$-separable strong generating set. The sifting of $g \in S_{n}$ by $H$ attempts to write $g$ as an element of $H$, and can be used for membership testing. For more details on sifting, please refer to [Ser03, Chapter 4].

Definition 3.13. Let $B=\left[\beta_{1}, \beta_{2}, \ldots, \beta_{m}\right]$ be a base of $H$, and let $H^{[i]}$ for $1 \leq i \leq m+1$ be the stabilisers in the stabiliser chain defined by $B$, as in Definition 3.6. For each $1 \leq i \leq m$, let $R_{i}$ be a transversal of $H^{[i+1]}$ in $H^{[i]}$. Let $g \in S_{n}$ be given. The so-called sifting of $g$ by $H$ works as follows. We initialise $g_{1}:=g$. For $1 \leq i \leq m$, we recursively find $r_{i} \in R_{i}$ such that $\beta_{i}^{r_{i}}=\beta_{i}^{g_{i}}$, and set $g_{i+1}:=g_{i} r_{i}^{-1}$. The procedure terminates when either

1. $2 \leq s \leq m$ and there are no $r_{s} \in R_{s}$ such that $\beta_{s}^{r_{s}}=\beta_{s}^{g_{s}}$, or

2. $s=m+1$ and we have computed $g_{s}$.

In both cases, $g_{s}$ is a siftee of $g$ by $H$.

We may conclude that $g \in H$ if $g_{m+1}=g r_{1}^{-1} r_{2}^{-1} \ldots r_{m}^{-1}=1$. Observe that for each $1 \leq i \leq s-1$, the permutation $g_{i+1}$ fixes $\beta_{i}$. Then as $\beta_{i}$ is fixed by all $R_{j}$ for $j \geq i$, the siftee $g_{s}$ also fixes $\beta_{i}$. Note also that $g_{s}$ is a product of $g$ and an element of $H$ :

Lemma 3.14. Let $g \in S_{n}$ and let $g^{\prime}$ be a siftee of $g$ by $H$. Then there exists $h \in H$ such that $g=g^{\prime} h$.

Proof. Let $B$ and the $R_{i}$ be as in Definition 3.13. Then there exist $1 \leq s \leq m+1$ and $r_{i} \in R_{i}$ for all $1 \leq i \leq s-1$ such that $g^{\prime}=g r_{1}^{-1} r_{2}^{-1} \ldots r_{s-1}^{-1}$. Since the $R_{i} \subseteq H$, the result follows.

Example 3.15 (sifting). Let $X=\{(1,2,3,4,5),(2,5)(3,4)\}$ and $H:=\langle X\rangle$. Then $X$ is a strong generating set of $H$ with respect to base $B:=[1,2]$. Let $R_{1}:=\{1,(1,5,4,3,2)$, $(1,4,2,5,3),(1,2)(3,5),(1,3,5,2,4)\}$ and $R_{2}:=\{1,(2,5)(3,4)\}$ be the transversals for $H_{(1)}$ in $H$ and for $H_{(1,2)}$ in $H_{(1)}$ respectively. Consider sifting $g=(1,2,4,5)$ by $H$. Initialise $g_{1}:=g$. Then $r_{1}:=(1,2)(3,5)$ is an element of $R_{1}$ mapping 1 to $1^{g_{1}}=2$. So $g_{2}=g_{1} r_{1}^{-1}=(2,4,3,5)$. Now there is no $r_{2} \in R_{2}$ mapping 2 to $2^{g_{2}}=4$. Therefore we get a siftee $(2,4,3,5)$.

If we have $(1,2,3,4,5)$ in $R_{1}$ instead of $(1,2)(3,5)$, then we get siftee $g(1,2,3,4,5)^{-1}=(2,3)$. 
The siftee of $g \in S_{n}$ by $H$ obtained from a sifting procedure is not unique: it depends on the choice of the base $B$ and the transversals $R_{i}$ associated with the stabiliser chain defined by $B$.

In our algorithm, we will be sifting elements of $H$ by stabilisers $H_{\left(\Delta_{i}\right)}$. Let $B:=\left(\beta_{1}, \ldots, \beta_{m}\right)$ be an orbit-ordered base of $H$ with respect to the ordering $\Omega_{1}, \Omega_{2}, \ldots, \Omega_{k}$. Then there exists $1 \leq j \leq m$ such that $\left(\beta_{j+1}, \beta_{j+2}, \ldots, \beta_{m}\right)$ is a base of $H_{\left(\Delta_{i}\right)}$ (where $j$ here is the $j_{i}$ from Definition 3.7), with associated stabiliser chain $H^{[j+1]} \geq H^{[j+2]} \geq \ldots \geq H^{[m+1]}$, which is a subchain of the stabiliser chain of $H$ defined by $B$. Whenever we sift an element $h \in H$ by a stabiliser $H_{\left(\Delta_{i}\right)}$, we sift using the partial stabiliser chain defined by an orbit-ordered base $B$ of $H$.

Definition 3.16. Let $B:=\left(\beta_{1}, \beta_{2}, \ldots, \beta_{m}\right)$ be an orbit-ordered base of $H$ and let $1 \leq j \leq m$ such that $\left(\beta_{j+1}, \beta_{j+2}, \ldots, \beta_{m}\right)$ is a base of $H_{\left(\Delta_{i}\right)}$. For $h \in H$, a siftee of $h$ by $H_{\left(\Delta_{i}\right)}$ is a siftee obtained by sifting $h$ using the stabiliser chain defined by the base $\left(\beta_{j+1}, \beta_{j+2}, \ldots, \beta_{m}\right)$.

For $x \in X$, we will be deciding whether $\rho_{i+1}(x) \in N_{i+1}$ by considering a siftee of $x$ by $H_{\left(\Delta_{i}\right)}$.

Lemma 3.17. Let $B$ be an orbit-ordered base of $H \leq S_{n}$ with respect to the $H$-orbits ordering $\Omega_{1}, \Omega_{2}, \ldots, \Omega_{k}$. Let $x \in H$ and $x^{\prime}$ be a siftee of $x$ by $H_{\left(\Delta_{i}\right)}$, where we sift $x$ as in Definition 3.16. Then $\rho_{i+1}(x) \in N_{i+1}$ if and only if $\rho_{i+1}\left(x^{\prime}\right)=1$.

Proof. If $\rho_{i+1}(x) \in N_{i+1}$, by Lemma 3.14, there exists $h \in H_{\left(\Delta_{i}\right)}$ such that $x=x^{\prime} h$. So $\rho_{i+1}(x)=\rho_{i+1}\left(x^{\prime}\right) \rho_{i+1}(h) \in N_{i+1}$.

Suppose now that $\rho_{i+1}\left(x^{\prime}\right)=1$. Since $B$ is orbit-ordered, there exist $1 \leq t \leq u \leq m$ such that $H_{\left(\beta_{1}, \beta_{2}, \ldots, \beta_{t}\right)}=H_{\left(\Delta_{i}\right)}$ and $H_{\left(\beta_{1}, \beta_{2}, \ldots, \beta_{u}\right)}=H_{\left(\Delta_{i+1}\right)}$. So $\left[\beta_{t+1}, \beta_{t+2}, \ldots, \beta_{u}\right]$ is a base of $\rho_{i+1}\left(H_{\left(\Delta_{i}\right)}\right)=N_{i+1}$. Since $\rho_{i+1}(x) \in N_{i+1}$, sifting $x$ does not terminate until after we have considered the image of $\beta_{u}$. More specifically, there exists $u \leq s \leq m+1$ and $r_{i} \in R_{i}$ for all $1 \leq i \leq s-1$ such that $x^{\prime}=x r_{t+1}^{-1} r_{t+2}^{-1} \ldots r_{s-1}^{-1}$. Hence $x^{\prime}$ fixes $\left[\beta_{t+1}, \beta_{t+1}, \ldots, \beta_{u}\right]$. Therefore $\rho_{i+1}\left(x^{\prime}\right)$ is an element of $N_{i+1}$ fixing the base of $N_{i+1}$, so $\rho_{i+1}\left(x^{\prime}\right)=1$.

\section{Algorithm}

In this section, we present an algorithm to compute the finest disjoint direct product decomposition of a given permutation group $H=\langle X\rangle \leq S_{n}$ and show that it has polynomial time complexity in terms of $n \cdot|X|$.

Recall Notation 2.4. For $1 \leq i \leq k$, let $\mathcal{P}_{i}$ be as in Definition 3.3. As the base case, we begin with $\mathcal{P}_{1}:=\langle\{1\}\rangle$ and we will compute $\mathcal{P}_{i+1}$ iteratively using Proposition 3.4.

Our algorithm to compute the finest disjoint direct product decomposition is presented in Algorithm 1. This algorithm computes a base and an initial strong generating set for $H$ and then calls Algorithm 2 repeatedly to calculate the $\mathcal{P}_{i}$.

We give an example for Algorithm 2 here.

Example 4.1 (running example). Consider $H$ in Example 3.11. We have seen that $\mathcal{P}_{2}=$ $\langle\{1\} \mid\{2\}\rangle$ and $X$ is 2-separable. To compute $\mathcal{P}_{3}$, initialise $S:=\{\}$. Observe that $P_{\overline{2}}\left(x_{1}\right) \in$ $P_{1}(H) \times 1_{2}$ and $x_{1} \notin H_{\left(\Delta_{2}\right)}$. Sifting $x_{1}$ by $H_{\left(\Delta_{2}\right)}$ gives us a siftee $(1,2,3)$. Similarly, $P_{\overline{2}}\left(x_{2}\right) \in$ $P_{2}(H) \times 1_{1}$ and $x_{2} \notin H_{\left(\Delta_{2}\right)}$ with a siftee $(4,5,6)$. Now $x_{3} \in P_{2}(H) \times 1_{1}$ and $x_{3} \notin H_{\left(\Delta_{2}\right)}$ has a siftee $(5,6)(8,9)(11,12)$, which has a non-trivial projection on $\{7,8,9\}$. So we add $\{2\}$ to 

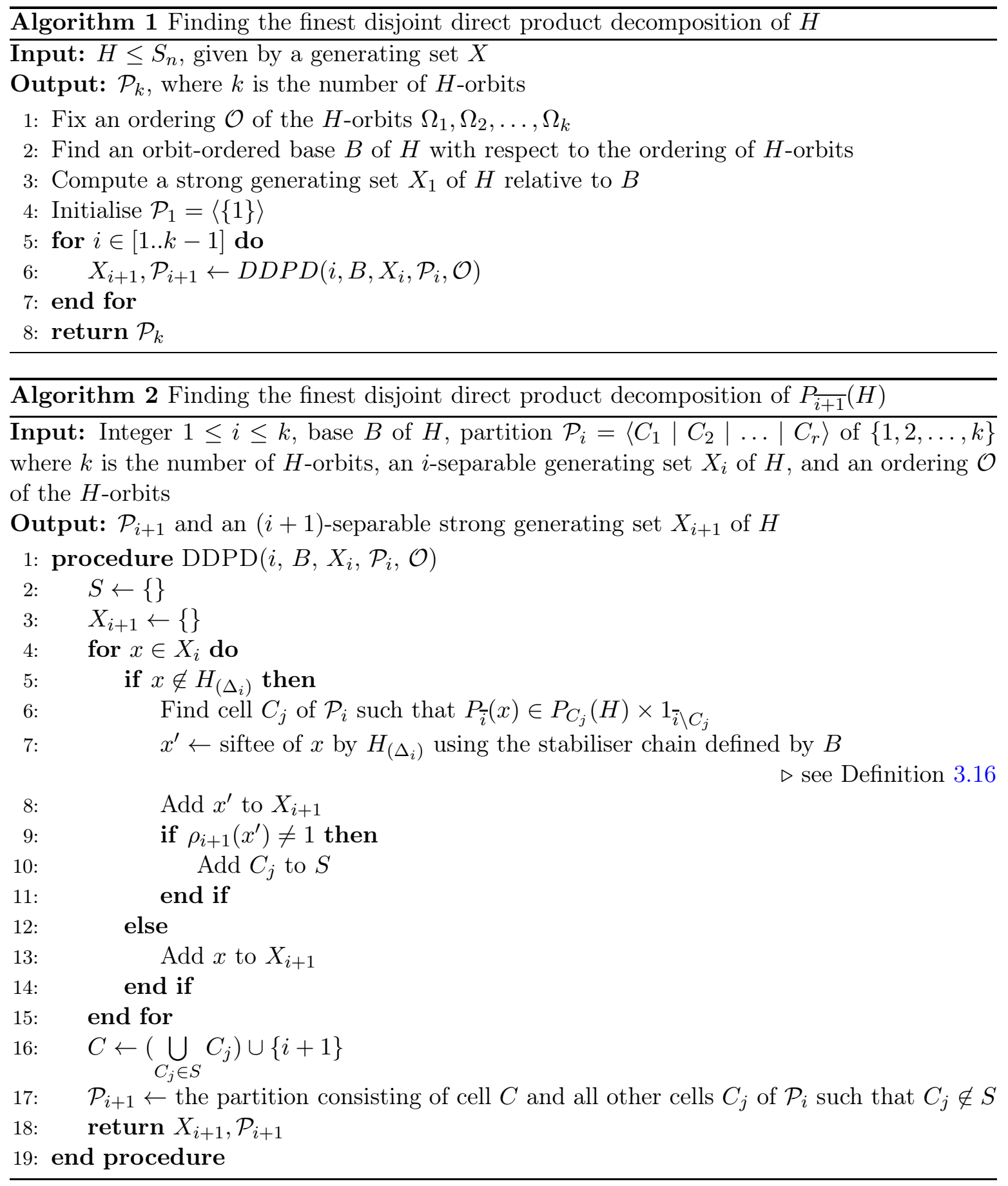

S. Since $x_{4} \in H_{\left(\Delta_{2}\right)}$ we add $x_{4}$ to $X_{3}$. Therefore, $X_{3}=\{(1,2,3),(4,5,6),(5,6)(8,9)(11,12)$, $(7,8,9)(10,11,12)\}$ and $\mathcal{P}_{3}=\langle\{1\} \mid\{2,3\}\rangle$.

Lemma 4.2. For $1 \leq i \leq k$, the sets $X_{i}$ computed in Algorithms 1 and 2 are strong generating sets of $H$ with respect to the base $B=\left[\beta_{1}, \beta_{2}, \ldots, \beta_{m}\right]$ defined on line 2 of Algorithm 1.

Proof. We proceed by induction on $i$. Clearly $X_{1}$ is a strong generating set of $H$ with respect to $B$. Suppose that $X_{i}$ is a strong generating set of $H$ with respect to $B$. First note that since 
each $x \in X_{i} \cap H_{\left(\Delta_{i}\right)}$ is also in $X_{i+1}$ and a siftee of $x \in X_{i} \backslash H_{\left(\Delta_{i}\right)}$ by $H_{\left(\Delta_{i}\right)}$ is not in $H_{\left(\Delta_{i}\right)}$, we have $X_{i} \cap H_{\left(\Delta_{i}\right)}=X_{i+1} \cap H_{\left(\Delta_{i}\right)}$. Let $0 \leq s \leq m$ and consider $H^{[s+1]}=H_{\left(\beta_{1}, \beta_{2}, \ldots, \beta_{s}\right)}$. Since $B$ is an orbit-ordered base, either $H^{[s+1]} \subseteq H_{\left(\Delta_{i}\right)}$ or $H_{\left(\Delta_{i}\right)} \subseteq H^{[s+1]}$. For both cases, we will show that $H^{[s+1]}=\left\langle X_{i+1} \cap H^{[s+1]}\right\rangle$.

Suppose first that $H^{[s+1]} \subseteq H_{\left(\Delta_{i}\right)}$. Then

$$
X_{i} \cap H^{[s+1]}=X_{i} \cap H_{\left(\Delta_{i}\right)} \cap H^{[s+1]}=X_{i+1} \cap H_{\left(\Delta_{i}\right)} \cap H^{[s+1]}=X_{i+1} \cap H^{[s+1]} .
$$

Since $X_{i}$ is a strong generating set, we have $H^{[s+1]}=\left\langle X_{i} \cap H^{[s+1]}\right\rangle=\left\langle X_{i+1} \cap H^{[s+1]}\right\rangle$.

Suppose instead that $H_{\left(\Delta_{i}\right)} \subseteq H^{[s+1]}$. Certainly $\left\langle X_{i+1} \cap H^{[s+1]}\right\rangle \leq H^{[s+1]}$. To show the reverse containment, since $X_{i}$ is a strong generating set, it suffices to show that $X_{i} \cap H^{[s+1]} \subseteq$ $\left\langle X_{i+1} \cap H^{[s+1]}\right\rangle$. Let $x \in X_{i} \cap H^{[s+1]}$. If $x \in H_{\left(\Delta_{i}\right)}$, then by the construction of $X_{i+1}$, we have $x \in X_{i+1}$, hence $x \in X_{i+1} \cap H^{[s+1]}$. Else suppose that $x \in\left(X_{i} \cap H^{[s+1]}\right) \backslash H_{\left(\Delta_{i}\right)}$. Then there exists a siftee $x^{\prime}$ of $x$ by $H_{\left(\Delta_{i}\right)}$ such that $x^{\prime} \in X_{i+1}$. By Lemma 3.14, there exists $g \in H_{\left(\Delta_{i}\right)} \leq H^{[s+1]}$ such that $x=x^{\prime} g$. Then $x^{\prime}=x g^{-1} \in H^{[s+1]}$ and so $x^{\prime} \in X_{i+1} \cap H^{[s+1]}$. Since

$$
g \in H_{\left(\Delta_{i}\right)}=\left\langle X_{i} \cap H_{\left(\Delta_{i}\right)}\right\rangle=\left\langle X_{i+1} \cap H_{\left(\Delta_{i}\right)}\right\rangle \subseteq\left\langle X_{i+1} \cap H^{[s+1]}\right\rangle,
$$

we have $x \in\left\langle X_{i+1} \cap H^{[s+1]}\right\rangle$.

Lemma 4.3. Let $1 \leq i \leq k-1$. Let $X_{i}$ be an $i$-separable generating set of $H$ and let $\mathcal{P}_{i}=\left\langle C_{1}\right|$ $\left.C_{2}|\ldots| C_{r}\right\rangle$ be as in Definition 3.3. Let $X_{i+1}$ and $Q$ be the output of $D D P D\left(i, B, X_{i}, \mathcal{P}_{i}\right)$ from Algorithm 2. Then

1. $\mathcal{P}_{i+1}=Q$.

2. $X_{i+1}$ is an $(i+1)$-separable generating set of $H$.

Hence Algorithm 2 is correct.

Proof. Item 1: We show that the set $S$ constructed from Algorithm 2 is the same as the set in Proposition 3.4, from which the result will follow. Observe that we add $C_{j}$ to $S$ in line 10 if and only if there exists $x \in X_{i}$ with non-trivial projection $P_{\bar{i}}(x)$ such that $P_{\bar{i}}(x) \in P_{C_{j}}(H) \times 1_{\bar{i} \backslash C_{j}}$ and $\rho_{i+1}\left(x^{\prime}\right) \neq 1$, where $x^{\prime}$ is a siftee of $x$ by $H_{\left(\Delta_{i}\right)}$. Since $X_{i}$ is $i$-separable, by Lemmas 3.12 and 3.17, such an $x \in X_{i}$ exists if and only if $\theta_{i}\left(P_{C_{j}}(H) \times 1_{\bar{i} \backslash C_{j}}\right) \neq N_{i+1}$. The result then follows from Proposition 3.4.

Item 2: Let $y \in X_{i+1}$. We show that if $P_{\overline{i+1}}(y) \neq 1$, then there exists a unique cell $C^{\prime}$ of $\mathcal{P}_{i+1}$ such that $P_{\overline{i+1}}(y) \in P_{C^{\prime}}(H) \times 1 \overline{i+1} \backslash C^{\prime}$.

By the construction of $X_{i+1}$, either $y \in X_{i} \cap H_{\left(\Delta_{i}\right)}$ or there exists $x \in X_{i} \backslash H_{\left(\Delta_{i}\right)}$ such that $y$ is a siftee of $x$ by $H_{\left(\Delta_{i}\right)}$. Suppose first that $y \in X_{i} \cap H_{\left(\Delta_{i}\right)}$, so $P_{\bar{i}}(y)=1$. If $P_{\overline{i+1}}(y) \neq 1$, then $\rho_{i+1}(y) \neq 1$ and so $P_{C}(y) \neq 1$, where $C$ is defined as in line 16 . Since $\overline{i+1} \backslash C=\bar{i} \backslash C \subseteq \bar{i}$, we have $P_{\overline{i+1} \backslash C}(y)=1$. So $C$ is the unique cell of $\mathcal{P}_{i+1}$ such that $P_{\overline{i+1}}(y)=P_{C}(y) \times 1 \overline{\overline{i+1} \backslash C} \in$ $P_{C}(H) \times 1 \overline{i+1} \backslash C$.

Suppose now that $y$ is a siftee of some $x \in X_{i} \backslash H_{\left(\Delta_{i}\right)}$ by $H_{\left(\Delta_{i}\right)}$. By Lemma 3.14, there exists $g \in H_{\left(\Delta_{i}\right)}$ such that $x=y g$. Then since $P_{\bar{i}}(g)=1$, we have $P_{\bar{i}}(x)=P_{\bar{i}}(y)$. As $X_{i}$ is $i$ separable, there exists exactly one cell $C_{j}$ of $\mathcal{P}_{i}$ such that $P_{\bar{i}}(y)=P_{\bar{i}}(x) \in P_{C_{j}}(H) \times 1_{\bar{i} \backslash C_{j}}$.

If $C_{j} \notin S$, then $C_{j}$ is a cell of $\mathcal{P}_{i+1}$ and so $P_{\overline{i+1}}(y) \in P_{C_{j}}(H) \times 1 \overline{i+1} \backslash C_{j}$. Otherwise if $C_{j} \in S$, then $P_{\overline{i+1}}(y) \in P_{C_{j} \cup\{i+1\}}(H) \times 1_{\bar{i} \backslash C_{j}}$. In particular, since $C_{j} \cup\{i+1\} \subseteq C$, we have $P_{\overline{i+1}}(y) \in P_{C}(H) \times 1 \overline{i+1} \backslash C$. 
The following results are fundamental in permutation group algorithms. For more information, refer to, for example, [Ser03, HEO05].

Lemma 4.4. Given $K \leq S_{n}$ by a generating set $Y$, the following permutation group algorithms run in time polynomial in $n \cdot|Y|$ :

1. Computing orbits of $K$.

2. Computing a strong generating set $X$ of $K$ with respect to a given base $B$.

3. Computing pointwise stabilisers of $K$.

4. Obtaining a siftee of $x \in S_{n}$ by $K$ using the sifting procedure.

5. Testing membership of $x \in S_{n}$ in $K$.

Theorem 4.5. Given $H \leq S_{n}$ by the generating set $X$, the finest disjoint direct product decomposition of $H$ can be computed in time polynomial in $n \cdot|X|$.

Proof. Let $Q:=\left\langle C_{1}\left|C_{2}\right| \ldots \mid C_{r}\right\rangle$ be the partition computed by Algorithm 1. Then by Item 1 of Lemma $4.3, Q=\mathcal{P}_{k}$, and so $H=P_{C_{1}}(H) \times P_{C_{2}}(H) \times \ldots \times P_{C_{r}}(H)$ is the finest disjoint direct product decomposition of $H$.

It remains to show that Algorithm 1 runs in polynomial time. By Lemma 4.4, lines 1 and 3 run in polynomial time. Furthermore $\left|X_{1}\right|$ is of polynomial size. By Remark 3.8, we can get an orbit-ordered base in polynomial time. Since Algorithm 2 is called at most $O(n)$ times, it suffices to show that Algorithm 2 runs in time polynomial in $n \cdot|X|$.

By Lemma 4.4, lines 5 and 7 can be done in polynomial time. Line 6 can be done by iterating through each cell $C_{j}$ of $\mathcal{P}_{i}$ and checking if $P_{C_{j}}(x) \neq 1$. Since $\mathcal{P}_{i}$ has at most $k$ cells, this can be done in $O(k)$ time. Lastly since $\left|X_{i+1}\right|=\left|X_{i}\right|$, the result follows.

\section{Experiments}

In this section, we will investigate the practical performance of our algorithm for finding the finest disjoint direct product decomposition of a permutation group. As well as illustrating the performance of our algorithm, we will show how it can be used to improve the performance of a range of GAP implementations to solve important group-theoretic problems. Our algorithm is implemented in GAP 4.11 [GAP20].

We will test our algorithm on randomly created groups. The creator we use in this paper is very straightforward and does not claim to produce all groups with equal probability. The creator takes three parameters, a transitive permutation group $G$ and two integer constants $r$ and $s$. The algorithm will produce a permutation group which is the direct product of $r$ groups. Each of these direct factors will be a subdirect product of $G^{s}$ that is d.d.p. indecomposable. The algorithm runs in two stages.

The first stage is implemented by a function MAKeSubdireCt $(G, s)$ which produces a random subdirect product of $s$ copies of $G$ that is d.d.p. indecomposable. $\operatorname{Makesubdirect}(G, s)$ works by taking a random integer $i \in\{2,3, \ldots, s\}$ and then taking the group $H$ generated by $i$ random elements of $G^{s}$. If $H$ is d.d.p. indecomposable, and its projection onto each of the $s$ copies of $G$ is surjective, then it is returned, else this procedure repeats.

The second stage simply runs MAKeSubdirect $(G, s) r$ times, and takes the direct product of these $r$ groups. Finally, we conjugate this group by a random permutation on the set of 
points moved by this group, so the decomposition does not follow the natural ordering of the integers.

Our algorithmic section is split into two sections. Firstly we compare our algorithm against the algorithm of Donaldson and Miller [DM09]. Secondly, to demonstrate that our algorithm has immediate practical value, we show how a few functions in GAP can easily be sped up by the knowledge of a disjoint direct product decomposition.

\subsection{Comparison to Donaldson and Miller}

We first compare to Donaldson and Miller's algorithm. Donaldson and Miller present two algorithms, an incomplete algorithm and a complete algorithm. We will not compare against their incomplete algorithm, as it is extremely fast but requires separable strong generating sets. Donaldson and Miller were unable to find a graph where the generating set of the automorphism group produced by nauty [MP14] is not separable. We were able to find graphs where nauty does not produce a separable generating set ${ }^{2}$. Also, the most advanced graph automorphism finders, such as Traces [MP14], perform random dives which produce random automorphisms. This means generating sets produced by Traces will not, in general, be separable.

When implementing Donaldson and Miller's complete algorithm, we were forced to make some implementation choices. Most significant is the ordering in which the algorithm tries to partition the orbits (the first line of Algorithm 5 in [DM09]). We implemented this by trying the partitions in order of increasing size of the smaller part of the partition. This has the advantage that when the finest disjoint direct product decomposition has a factor with few orbits the algorithm will run relatively quickly - it does not affect the algorithm's worst case complexity.

We gather our results in Figure 1. Each row of Figure 1 gives the result for 10 instances of $H$, created as above. Each instance of $H$ has $r s$ orbits with the finest disjoint direct product decomposition consisting of $r$ direct factors with $s$ orbits each, and each transitive constituent of $H$ is permutation isomorphic to $G$. For each $G, r$ and $s$, we report the median time ("Median") and the number of completed instances ("\#") of the 10 instances of $H$, using both Algorithm 5 of [DM09] ("Donaldson") and Algorithm 1 ("Our Algorithm"). The results are consistent with the theory. Since our algorithm is polynomial, it scales much better than the algorithm in [DM09].

\subsection{Application to GAP}

In this section, we will see how some functions in GAP can easily be sped up by the knowledge of a disjoint direct product decomposition. We do not claim that this is an exhaustive list, but we intend to demonstrate how, for a selection of common problems, calculating a disjoint direct product decomposition can significantly improve performance. We experimented with three GAP functions:

DerivedSubgroup computes the derived subgroup of a given group. The derived subgroup of a group $H$ is the direct product of the derived subgroup of each disjoint direct factor of $H$.

\footnotetext{
${ }^{2}$ Some examples and how one could look for more examples can be found in the supplemental files.
} 


\begin{tabular}{ccccccc}
\hline $\mathrm{G}$ & $\mathrm{r}$ & $\mathrm{s}$ & \multicolumn{2}{c}{ Donaldson } & \multicolumn{2}{c}{ Our Algorithm } \\
& & & Median & $\#$ & Median & $\#$ \\
\hline$D_{8}$ & 4 & 4 & 0.04 & 10 & 0.00 & 10 \\
$D_{8}$ & 6 & 4 & 0.16 & 10 & 0.01 & 10 \\
$D_{8}$ & 8 & 4 & 0.54 & 10 & 0.01 & 10 \\
$D_{8}$ & 10 & 4 & 2.18 & 10 & 0.02 & 10 \\
\hline$A_{4}$ & 4 & 4 & 2.47 & 10 & 0.01 & 10 \\
$A_{4}$ & 6 & 4 & 12.47 & 10 & 0.03 & 10 \\
$A_{4}$ & 8 & 4 & 158.09 & 10 & 0.04 & 10 \\
$A_{4}$ & 10 & 4 & 490.54 & 5 & 0.05 & 10 \\
\hline$S_{4}$ & 4 & 4 & 3.37 & 10 & 0.02 & 10 \\
$S_{4}$ & 6 & 4 & 15.99 & 10 & 0.04 & 10 \\
$S_{4}$ & 8 & 4 & 393.86 & 8 & 0.06 & 10 \\
$S_{4}$ & 10 & 4 & $\mathrm{~N} / \mathrm{A}$ & 0 & 0.07 & 10 \\
\hline
\end{tabular}

Figure 1: Comparison of DDPD algorithms

NrConjugacyClasses gives the number of conjugacy classes of a given group. The number of conjugacy classes of a group $H$ is the product of the number of conjugacy classes of each disjoint direct factor of $H$.

CompositionSeries computes a composition series of a given group. A composition series of a group $H$ can be constructed from composition series of the direct factors of $H$.

In our experiments, we run each row of our tables ten times. Each run is given a limit of 10 minutes and $4 \mathrm{~GB}$ of memory. We give the median time in seconds (or N/A when less than 6 instances finished successfully). For the inner group $G$ we consider the alternating group $\left(A_{n}\right)$, symmetric group $\left(S_{n}\right)$, dihedral groups $\left(D_{2 n}\right)$ of varying degree $n$, and also TransitiveGroup $(16,712)$ (Trans $(16,712)$ ) and TransitiveGroup $(16,713)$ (Trans $(16,713))$ from the Transitive Groups Library [Hul17].

Each row of the tables in Figures 2 to 4 gives the results for 10 random groups $H$, each with $r s$ orbits, where the projection of $H$ onto each orbit is permutation isomorphic to $G$, and $H$ has the finest disjoint direct product decomposition consisting of $r$ direct factors with $s$ orbits each. The columns "Full Group" and "Decomposed Group" refer to the computation with the original group $H$ and the computation with the disjoint direct product factors of $H$ respectively. The column "Decomposition" refers to the computation of the finest disjoint direct product decomposition of $H$. For each of these columns, we report the median time (in seconds) required to compute the specified problems of the 10 instances under the subcolumns "Median", and the number of instances completed within the time and memory limits under the subcolumns "\#".

The time taken to find the finest disjoint direct product decomposition and solve DERIVEDSubgroup (Figure 2) and NrConjugacyClasses (Figure 3) on the decomposed group are always faster than solving the problem on the original full group. In the case of DERIVEDSuBGROUP (Figure 2), we speed up performance by up to a factor of 10. In the case of NRConjuGaCyClasses (Figure 3) and CompositionSeries (Figure 4), we are able to solve problems that previously ran out of memory or time, in under a second. Almost the entire time taken by Decomposition is building an initial stabiliser chain with respect to an orbit-ordered base. 
For example, in the largest problem in Figure 2, building the stabiliser chain took 22.5 of the 23.7 seconds taken in total. The time taken to build a stabiliser chain with respect to an orbit-ordered base is, on average, no longer than the time taken to build a stabiliser chain using GAP's default strategy for base ordering.

\begin{tabular}{ccccccccc}
\hline G & r & s & \multicolumn{2}{c}{ Full Group } & \multicolumn{2}{c}{ Decomposed Group } & \multicolumn{2}{c}{ Decomposition } \\
& & & Median & $\#$ & Median & $\#$ & Median & $\#$ \\
\hline$A_{4}$ & 12 & 4 & 2.34 & 10 & 0.01 & 10 & 0.28 & 10 \\
$A_{4}$ & 16 & 4 & 7.53 & 10 & 0.01 & 10 & 0.66 & 10 \\
$A_{4}$ & 20 & 4 & 19.76 & 10 & 0.01 & 10 & 1.64 & 10 \\
\hline$D_{8}$ & 12 & 4 & 0.33 & 10 & 0.00 & 10 & 0.20 & 10 \\
$D_{8}$ & 16 & 4 & 1.07 & 10 & 0.00 & 10 & 0.55 & 10 \\
$D_{8}$ & 20 & 4 & 2.53 & 10 & 0.00 & 10 & 1.20 & 10 \\
\hline$S_{4}$ & 12 & 4 & 7.36 & 10 & 0.01 & 10 & 0.89 & 10 \\
$S_{4}$ & 16 & 4 & 27.89 & 10 & 0.02 & 10 & 2.34 & 10 \\
$S_{4}$ & 20 & 4 & 65.85 & 10 & 0.02 & 10 & 5.37 & 10 \\
\hline$D_{32}$ & 12 & 4 & 6.58 & 10 & 0.01 & 10 & 1.02 & 10 \\
$D_{32}$ & 16 & 4 & 18.47 & 10 & 0.01 & 10 & 2.86 & 10 \\
$D_{32}$ & 20 & 4 & 44.72 & 10 & 0.02 & 10 & 6.47 & 10 \\
\hline Trans $(16,712)$ & 12 & 4 & 17.34 & 10 & 0.03 & 10 & 4.46 & 10 \\
Trans $(16,712)$ & 16 & 4 & 56.20 & 10 & 0.04 & 10 & 11.61 & 10 \\
Trans $(16,712)$ & 20 & 4 & 144.37 & 10 & 0.05 & 10 & 27.43 & 10 \\
\hline Trans $(16,713)$ & 12 & 4 & 13.53 & 10 & 0.03 & 10 & 3.69 & 10 \\
Trans $(16,713)$ & 16 & 4 & 38.17 & 10 & 0.05 & 10 & 10.80 & 10 \\
Trans $(16,713)$ & 20 & 4 & 90.89 & 10 & 0.06 & 10 & 23.72 & 10 \\
\hline
\end{tabular}

Figure 2: Performance of DDPD for DeRIVEdSubgroup

\begin{tabular}{ccccccccc}
\hline $\mathrm{G}$ & $\mathrm{r}$ & $\mathrm{s}$ & \multicolumn{2}{c}{ Full Group } & \multicolumn{2}{c}{ Decomposed Group } & \multicolumn{2}{c}{ Decomposition } \\
& & & Median & $\#$ & Median & $\#$ & Median & $\#$ \\
\hline$A_{3}$ & 2 & 4 & 0.00 & 10 & 0.00 & 10 & 0.00 & 10 \\
$A_{3}$ & 4 & 4 & 0.08 & 10 & 0.00 & 10 & 0.00 & 10 \\
$A_{3}$ & 6 & 4 & 21.50 & 6 & 0.00 & 10 & 0.00 & 10 \\
\hline$D_{8}$ & 2 & 4 & 0.23 & 10 & 0.00 & 10 & 0.00 & 10 \\
$D_{8}$ & 4 & 4 & $\mathrm{~N} / \mathrm{A}$ & 2 & 0.00 & 10 & 0.00 & 10 \\
$D_{8}$ & 6 & 4 & $\mathrm{~N} / \mathrm{A}$ & 0 & 0.01 & 10 & 0.00 & 10 \\
\hline$S_{4}$ & 2 & 4 & 50.52 & 10 & 0.28 & 10 & 0.00 & 10 \\
$S_{4}$ & 4 & 4 & $\mathrm{~N} / \mathrm{A}$ & 0 & 0.58 & 10 & 0.00 & 10 \\
$S_{4}$ & 6 & 4 & $\mathrm{~N} / \mathrm{A}$ & 0 & 0.96 & 10 & 0.02 & 10 \\
\hline
\end{tabular}

Figure 3: Performance of DDPD for NRConjugacyClasses 


\begin{tabular}{ccccccccc}
\hline G & r & s & \multicolumn{2}{c}{ Full Group } & \multicolumn{2}{c}{ Decomposed Group } & \multicolumn{2}{c}{ Decomposition } \\
& & & Median & $\#$ & Median & $\#$ & Median & $\#$ \\
\hline$A_{4}$ & 4 & 3 & 0.00 & 10 & 0.00 & 10 & 0.00 & 10 \\
$A_{4}$ & 12 & 3 & 0.06 & 10 & 0.01 & 10 & 0.11 & 10 \\
$A_{4}$ & 20 & 3 & 0.22 & 10 & 0.02 & 10 & 0.68 & 10 \\
\hline$S_{4}$ & 4 & 3 & 0.01 & 10 & 0.00 & 10 & 0.00 & 10 \\
$S_{4}$ & 12 & 3 & 0.15 & 10 & 0.02 & 10 & 0.32 & 10 \\
$S_{4}$ & 20 & 3 & 0.49 & 10 & 0.03 & 10 & 1.70 & 10 \\
\hline$D_{8}$ & 4 & 3 & 0.00 & 10 & 0.00 & 10 & 0.00 & 10 \\
$D_{8}$ & 12 & 3 & 0.03 & 10 & 0.01 & 10 & 0.07 & 10 \\
$D_{8}$ & 20 & 3 & 0.10 & 10 & 0.02 & 10 & 0.40 & 10 \\
\hline$D_{32}$ & 4 & 3 & 0.01 & 10 & 0.00 & 10 & 0.00 & 10 \\
$D_{32}$ & 12 & 3 & 0.11 & 10 & 0.02 & 10 & 0.27 & 10 \\
$D_{32}$ & 20 & 3 & 0.45 & 10 & 0.04 & 10 & 1.87 & 10 \\
\hline Trans $(16,712)$ & 4 & 3 & 0.02 & 10 & 0.01 & 10 & 0.03 & 10 \\
Trans $(16,712)$ & 12 & 3 & 0.36 & 10 & 0.03 & 10 & 1.25 & 10 \\
Trans $(16,712)$ & 20 & 3 & 1.52 & 10 & 0.06 & 10 & 8.72 & 10 \\
\hline Trans $(16,713)$ & 4 & 3 & 0.64 & 10 & 0.02 & 10 & 0.03 & 10 \\
$\operatorname{Trans}(16,713)$ & 12 & 3 & 80.49 & 10 & 0.07 & 10 & 1.32 & 10 \\
Trans $(16,713)$ & 20 & 3 & N $/ \mathrm{A}$ & 0 & 0.12 & 10 & 8.57 & 10 \\
\hline
\end{tabular}

Figure 4: Performance of DDPD for CompositionSeRIES

\section{Conclusion and future work}

In this paper, we have shown that the finest disjoint direct product decomposition of a given group can be computed efficiently and can be used to speed up various permutation group problems. Moreover, as demonstrated in [DM09, Gra11], the disjoint direct product decomposition of a group has applications beyond computational group theory.

While we show the disjoint direct product decomposition can be extremely useful, we are not suggesting it to be employed as an initial subprocedure of solving all the problems we use in our experiments. This is because adding this subprocedure will impose additional computation time on all calls of the problem that could be a waste of time if the group is d.d.p. indecomposable. Using efficient heuristics to only add this subprocedure for some groups still has the same problem with the added cost and raises an issue of determining the heuristics. Therefore, we propose that the computation of the finest disjoint direct product decomposition be available in GAP as a function, and leave it up to the user to decide if the decomposition would help the problem on hand.

An obvious piece of future work is to determine other problems, group theoretic or otherwise, that can benefit from the knowledge of its disjoint direct product decomposition. For example, the first author has found applications in her work with computing normalisers and the second author in his work with graph isomorphisms. We believe that the disjoint direct product decomposition has more potential in groups arising from real world problems, as these are more likely to be highly intransitive. 


\section{Acknowledgement}

The authors would like to thank the reviewers, Colva Roney-Dougal and Wilf Wilson for their useful comments. The second author is supported by a Royal Society University Research Fellowship.

\section{References}

[BSZ15] Kristine Bauer, Debasis Sen, and Peter Zvengrowski. A generalized Goursat lemma. Tatra Mt. Math. Publ., 64:1-19, 2015.

[DM06] Alastair F. Donaldson and Alice Miller. Exact and approximate strategies for symmetry reduction in model checking. FM 2006: Formal Methods, pages 541$556,2006$.

[DM09] Alastair F. Donaldson and Alice Miller. On the constructive orbit problem. Ann. Math. Artif. Intell., 57(1):1-35, 2009.

[GAP20] The GAP Group. GAP - Groups, Algorithms, and Programming, Version 4.11.0, 2020.

[GJMRD09] Andrew Grayland, Chris Jefferson, Ian Miguel, and Colva M. Roney-Dougal. Minimal ordering constraints for some families of variable symmetries. Ann. Math. Artif. Intell., 57(1):75-102, 2009.

[Gou89] Édouard Goursat. Sur les substitutions orthogonales et les divisions régulières de l'espace. Annales scientifiques de l'École Normale Supérieure, 3e série, 6:9-102, 1889.

[GPP06] Ian P. Gent, Karen E. Petrie, and Jean-François Puget. Chapter 10 - Symmetry in constraint programming. In Francesca Rossi, Peter van Beek, and Toby Walsh, editors, Handbook of Constraint Programming, volume 2 of Foundations of Artificial Intelligence, pages 329 - 376. Elsevier, 2006.

[Gra11] Andrews Grayland. Automated static symmetry breaking in constraint satisfaction problems. $\mathrm{PhD}$ thesis, University of St Andrews, 2011.

[HEO05] Derek F. Holt, Bettina Eick, and Eamonn A. O'Brien. Handbook of computational group theory. Discrete Mathematics and its Applications (Boca Raton). Chapman \& Hall/CRC, Boca Raton, FL, 2005.

[Hul17] Alexander Hulpke. TransGrp, transitive groups library, Version 2.0.2. http://www.math.colostate.edu/ hulpke/transgrp, Nov 2017. GAP package.

[Hun74] Thomas W. Hungerford. Algebra. Holt, Rinehart and Winston, Inc., New YorkMontreal, Que.-London, 1974.

[KN09] Neeraj Kayal and Timur Nezhmetdinov. Factoring groups efficiently. In $A u$ tomata, languages and programming. Part I, volume 5555 of Lecture Notes in Comput. Sci., pages 585-596. Springer, Berlin, 2009. 
[MP14] Brendan D. McKay and Adolfo Piperno. Practical graph isomorphism, $\{$ II $\}$. Journal of Symbolic Computation, 60(0):94 - 112, 2014.

[PS18] Cheryl E. Praeger and Csaba Schneider. Permutation groups and Cartesian decompositions, volume 449 of London Mathematical Society Lecture Note Series. Cambridge University Press, Cambridge, 2018.

[Sch94] Roland Schmidt. Subgroup lattices of groups, volume 14 of De Gruyter Expositions in Mathematics. Walter de Gruyter \& Co., Berlin, 1994.

[Ser03] Ákos Seress. Permutation group algorithms, volume 152 of Cambridge Tracts in Mathematics. Cambridge University Press, Cambridge, 2003.

[Sim70] Charles C. Sims. Computational methods in the study of permutation groups. In Computational Problems in Abstract Algebra (Proc. Conf., Oxford, 1967), pages 169-183. Pergamon, Oxford, 1970.

[Sim71] Charles C. Sims. Computation with permutation groups. In Proceedings of the Second ACM Symposium on Symbolic and Algebraic Manipulation, SYMSAC '71, page 23-28, New York, NY, USA, 1971. Association for Computing Machinery.

[Wil12] James B. Wilson. Existence, algorithms, and asymptotics of direct product decompositions, I. Groups Complex. Cryptol., 4(1):33-72, 2012. 